Ann. Zootech., I977, 26 (4), 6I5-6I9.

\title{
Note \\ Effet défavorable du mélange des sexes dans l'engraissement des porcs en groupe
}

\author{
L. OLLIVIER
}

Avec la collaboration technique de Marie-Reine Langrors et J. Gruand

Station de Génétique quantitative et appliquée

Centre national de Recherches zootechniques, I.N.R.A. 78350 Jouy en Josas (France)

\begin{abstract}
Résumé
Des contrôles d'alimentation et de croissance, de 30 à $80 \mathrm{~kg}$ de poids vif, ont été effectués sur 320 loges de porcs Large White, de 1966 à 1976 au Centre expérimental de Sélection porcine de Rouillé (Vienne). Chaque loge était constituée de pores mâles eastrés et de fernelles, en proportion variable, l'effectif de chaque loge étant voisin de so animaux an total. L'alimentation était distribuée à volonté au nourrisseur. Trois variables, exprimées en moyenne par loge, ont été analysées : le gain moyen quotidien par porc, la consommation d'aliment par porc et par jour et l'indice de consommation. L'évolution de ces trois variables en fonction du pourcentage de mâles castrés dans la loge s'écarte notablement de celle qu'on peut prédire d'après des différences observées par ailleurs entre sexes, dans les mêmes conditions d'engraissement mais sur des loges constituées d'animaux de même sexe. Comme, à l'intérieur d'une même loge, ces différences entre sexes ne semblent pas modifiées, l'explication invoquée est un effet du mélange, affectant également les performances des deux sexes. Cet effet peut être évalué, pour un mélange à égalité de porcs des deux sexes, à une baisse de 6,7 p. cent de la vitesse de croissance, de $\mathrm{x}, 8 \mathrm{p}$. cent de la consommation et à une augmentation de $4 \mathrm{p}$. cent de l'indice de consommation, par rapport à l'engraissement en loges de porcs de même sexe. L'explication de ce phénomène serait à trouver dans le comportement social du porc en croissance.
\end{abstract}

Dans une expérience entreprise en I966 au Centre expérimental de Sélection porcine de Rouillé (Vienne), dont les résultats détaillés sont publiés par ailleurs (Olifivier, I977), des porcs mâles castrés et femelles ont été mélangés en proportion variable pour constituer des loges d'environ ro porcs au total et nourris à volonté au nourrisseur. La consommation totale d'aliment par loge, le nombre de 
jours en présence et le gain de poids total des porcs de la loge (y compris les porcs éliminés en cours de contrôle) étaient enregistrés sur une période allant du jour où le poids moyen des pores de la loge avoisinait $30 \mathrm{~kg}$ jusqu'au jour où le poids moyen (des porcs non éliminés) avoisinait $80 \mathrm{~kg}$. Chaque loge était ainsi caractérisée par 3 variables :

I) le gain moyen quotidien (gain de poids total/jours totaux de présence),

2) la consommation moyenne journalière (consommation totale/jours totaux de présence),

3) l'indice de consommation (gain de poids total/consommation totale).

Les données analysées dans cette note ont été recueillies de I966 à I976, soit 320 loges au total, réparties sur 32 combinaisons année-bâtiment, et représentant la descendance de II générations successives de verrats, soit une de plus que dans 1'article mentionné au début. Le même modèle linéaire additif que dans cet article a été appliqué et le programme général de moindres carrés de HARver (I972) a également été utilisé. Rappelons que cette analyse permet d'estimer l'effet du taux de masculinité qui nous intéresse ici (c'est-à-dire du pourcentage de mâles castrés dans la loge au début du contrôle) après élimination des facteurs bâtiment, génération du père, poids moyen initial, poids moyen final et nombre d'animaux sortis avant la fin du contrôle. Dans une première analyse, les valeurs du taux de masculinité ont été regroupées en dix classes, de o à 9 , de ro à I 9 , etc... Dans une deuxième analyse, le taux de masculinité a été considéré comme une variable continue et les régressions linéaires des 3 variables en fonction de ce taux ont été estimées.

Le graphique ci-contre montre le résultat de cette analyse quant à l'effet du taux de masculinité sur les trois variables considérées. A titre de comparaison, le même graphique montre l'évolution attendue des 3 variables en fonction du taux de masculinité, sur la base des différences entre sexes estimées par SELI,IER (I977) dans les mêmes conditions d'engraissement, mais sur des loges toujours constituées d'animaux de même sexe.

\section{TABLEAU I}

Différences entre sexes (mâles castrés-femelles) (Sex differences)

\begin{tabular}{|c|c|c|c|}
\hline & $\begin{array}{c}\text { Sex séparés } \\
\text { Sexes kept separate } \\
\text { (SHLIER, I977) } \\
(a)\end{array}$ & $\begin{array}{l}\text { Sexes mélangés } \\
\text { Sexes mixed } \\
\text { (présente note) } \\
(\text { b) (I) }\end{array}$ & $\begin{array}{c}\text { Effet du mélange } \\
\text { Effect of mixing } \\
(b-a)\end{array}$ \\
\hline $\begin{array}{l}\text { Gain moyen quotidien }(\mathrm{kg}) . . \\
\text { Average daily gain } \\
\text { Consommation moyenne journa- } \\
\text { lière (kg). . . . . . . . } \\
\text { Daily feed intake } \\
\text { Indice de consommation }(\mathrm{kg} \text { ali- } \\
\text { ment } / \mathrm{kg} \text { de gain) . . . . . . } \\
\text { Feed conversion ratio }\end{array}$ & $\begin{array}{l}0,29 \pm 0,07 \\
0,08 \pm 0,05\end{array}$ & $\begin{array}{r}-0,036 \pm 0,029 \\
0,2 I \pm 0,06 \\
0,35 \pm 0,08\end{array}$ & $\begin{array}{c}-0,089\left(^{* *}\right) \\
-0,08 \mathrm{NS} \\
0,27\left(^{* *}\right)\end{array}$ \\
\hline
\end{tabular}

(I) $b$ est le coefficient de régression en fonction du rapport nombre de mâles/nombre de mâles et femelies dans la loge. $\mathrm{b}$ is the regression coeficient on pen sex-ratio.

NS : Différence non significative. Difference not significant.

$\left.{ }^{* *}\right)$ : Différence significative au seuil de $x$ p. cent. Significant difference at the $I$ p. cent level. 

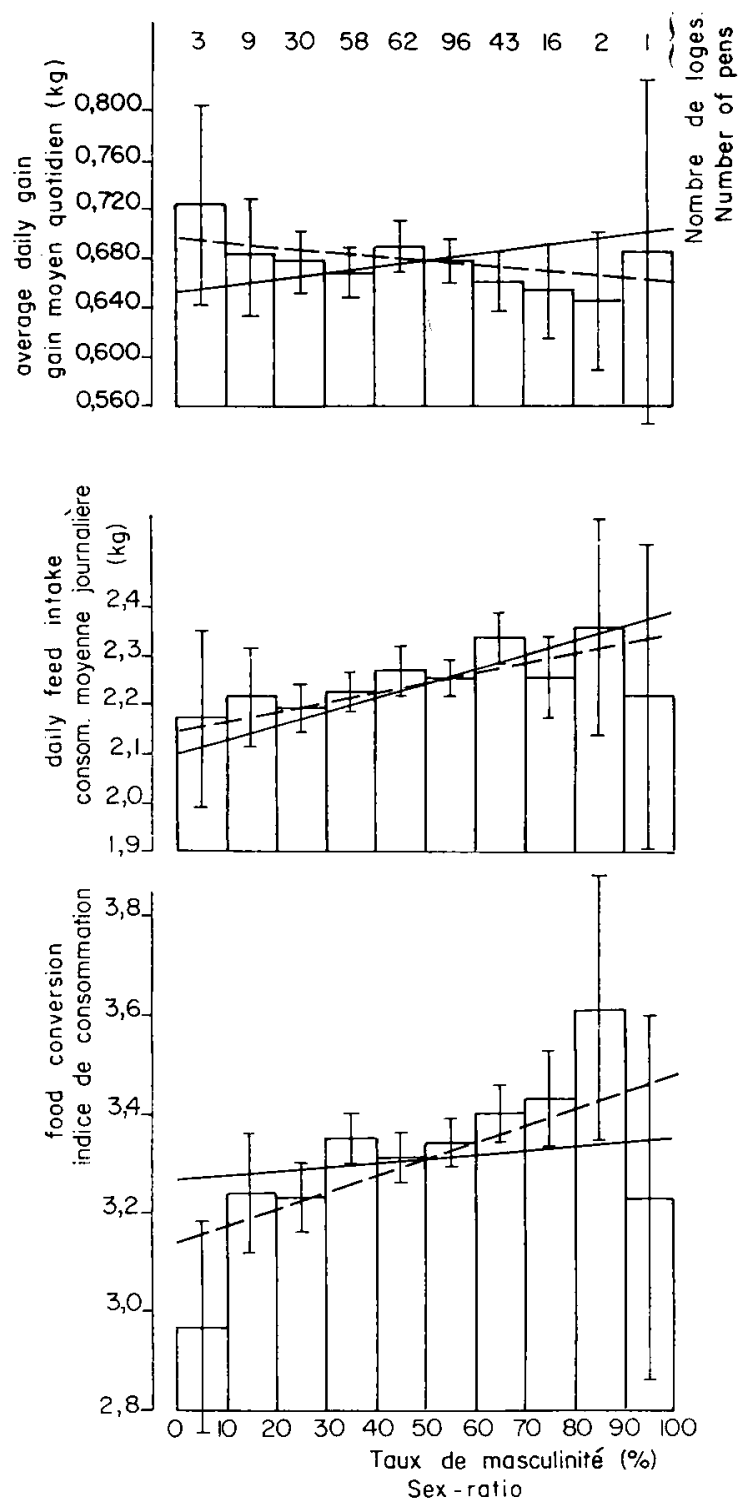

Vitesse de croissance, consommation d'aliment

et indice de consommation en fonction du taux de masculinité de la loge

(Growth rate, feed intake and food conversion ratio as a function of pen sex-ratio!)

Évolution en fonction des différences entre sexes (trendexpected from sex differences). Évolution observée (observed trend).

On voit que 1'effet du taux de masculinité est différent de celui ${ }_{\circ}^{\top} \mathrm{qu}^{\prime}$ 'on pourrait déduire de ces différences entre sexes, sauf peut-être pour la consommation journalière (tabl. I). Celle-ci augmente moins vite qu'on ne s'y attendrait en fonction du nombre de mâles dans la loge. L'indice de consommation par contre aug- 
mente nettement plus vite et le contraste est aussi très marqué pour la vitesse de croissance qui tend à diminuer quant le nombre de mâles augmente, alors que ceux-ci, lorsqu'ils sont engraissés séparément, ont une croissance plus rapide que les femelles.

Tout se passe, en apparence, comme si le mélange d'animaux de sexe différent modifiait les différences entre sexes qu'on observe avec l'alimentation en groupe de même sexe, ou en alimentation individuelle. Cette hypothèse, invérifiable pour la consommation qui n'est pas mesurée individuellement, semble devoir être écartée pour la vitesse de croissance. Car, sur les mêmes données, la différence, estimée sur la croissance individuelle de 30 à Ioo $\mathrm{kg}$, est de $4 \mathrm{I} \mathrm{g} /$ jour en faveur des mâles (OLIIVIER, I977). D'autre part, dans les stations danoises le passage d'une alimentation par groupe de 2 mâles castrés et 2 femelles à une alimentation individuelle s'est effectivement traduit par une inversion de la différence entre sexes pour la croissance, mais celle-ci était en faveur des mâles castrés avec l'alimentation en groupe (Jonsson, I959), ce qui va à l'encontre de l'hypothèse qui vient d'être avancée. Si on admet que les différences entre sexes sont inchangées, il faut alors supposer que le mélange d'animaux de sexe différent exerce par lui-même un effet sur les performances moyennes de la loge. Nos données ne permettent pas d'estimer rigoureusement l'importance du phénomène, car elles n'incluent aucune loge constituée uniquement de mâles ou de femelles. Cependant, en supposant que les régressions observées en fonction du taux de masculinité sont la résultante de deux effets linéaires, d'une part celui qui découle des différences entre sexes, d'autre part un effet propre du mélange, ce dernier peut être estimé par la différence entre les deux premières colonnes du tableau I. Le résultat, reporté dans la troisième colonne de ce tableau, doit être divisé par deux pour obtenir l'effet d'un mélange à égalité de porcs des deux sexes. Relativement aux moyennes de cette expérience, ce mélange entrâ̂ne une réduction de $6,7 \mathrm{p}$. cent de la vitesse de croissance, de I, 8 p. cent de la consommation d'aliment et un accroissement de $4 \mathrm{p}$. cent de l'indice de consommation. Autrement dit, ces valeurs représentent, pour un effectif comprenant $50 \mathrm{p}$. cent de porcs mâles et $50 \mathrm{p}$. cent de porcs femelles, le gain qu'apporte le regroupement par sexe par rapport à un mélange en proportion variable et aléatoire d'animaux de sexe différent.

Des effets similaires se dégagent des résultats de BeNkov VASILEV et STEFANOVA (I973) et de BEKAERT et CASTEELS (I976), qui comparent dans la même expérience des loges de porcs de même sexe et des loges contenant des porcs des deux sexes en nombre égal. Il apparaît que la vitesse de croissance des loges mixtes est inférieure à la moyenne des loges à sexes séparés et que l'indice de consommation des loges mixtes est supérieur à cette moyenne, l'effet défavorable du mélange atteignant près de $6 \mathrm{p}$. cent pour l'indice de consommation dans les essais des premiers auteurs.

L'explication du phénomène est sans doute à rechercher dans le comportement social du porc en croissance. Mc BRIDE JAMEs et HodGENs(I964) ont en effet montré que le rang social dans la loge explique 13 p. cent de la variabilité de croissance observée entre 2 et 4 mois d'âge, et ils indiquent aussi que, comme chez le poulet, le rang social chez les mâles est moins stable que chez les femelles. Cette instabilité pourrait expliquer les résultats que nous observons sur la vitesse de croissance, à condition de supposer que cette instabilité ne s'exprime pleinement qu'en présence de porcs de sexe femelle. 


\section{Summary \\ A note on the unfavourable effect on fattening traits of mixing female and castrated male pigs in the same pen}

Growth and feed intake, between 30 and $80 \mathrm{~kg}$ liveweight have been recorded on 320 pens of Large White pigs, from I966 to I976, at the INRA experimental farm of Rouille (Vienne). Each pen included a variable proportion of castrates and females over a total number of about ro pigs. These had free access to water :nd self-feeders. Growth rate, feed intake and feed efficiency have been analyzed, on a pen basis. The observed trends of these variables as a function of the pen sex-ratio markedly differ from those which could be expected from sex differences estimated under the same fattening conditions but on pens including only males or only females. As within pen these sex differences appear unchanged (at least for growth rate), the explanation put forward is an unfavourable per se effect of mixing together males and females, the two sexes being equally affected. On that basis, mixing the two sexes in the same pen (half and half) can be estimated to reduce growth rate by 6.7 per cent, feed intake by 1.8 per cent and to increase the feed conversion ratio by 4 percent, as compared to pens of like sex pigs. The social behaviour of the growing pig may be implied in this phenomenon.

\section{Références bibliographiques}

BEKAER'T H., CASTEELS M., I976. L'engraissement en groupes séparés de castrats et de truies du Landrace Belge et de la race Piétrain, avec application de l'alimentation à satiété et du rationnement. Rev. agric. (Bruxelles), 29, 96I-979.

Benkov M., VAsilev T., STEFANOVA S., 1973. Pig fattening as affected by their segregation by sex (en bulgare), Zhivotnovyd. Nauki, 10 (I), 89-92.

HARVEY W. R., 1972. Least-squares and maximum-likelihood general purpose program. Ohio State University, $3 \mathrm{I}$ p. (ronéoté).

Jonsson P., I959. Investigations on group feeding versus individual feeding and on the interaction between genotype and environment in pigs. Acta Agric., Scand., 9, 204-228.

Mc BRIDE G., James J. W., Hodgens N., I964. Social behaviour of domestic animals. IV. Growing pigs. Anim. Prod., 6, I29-1 39.

OLLIVIER L., I977. Dix ans d'une expérience de sélection individuelle sur des verrats utilisés en insémination artificielle. I. Réponses observées sur des caractères de croissance, de carcasse et de qualité de viande. Ann. Génét. Sél. anim., 9, 353-377.

SELIIER P., I977. Valeur en croisement de verrats Large White et Piétrain; influence du poids d'abattage. In: Journées de la Recherche Porcine en France, 85-89, Institut technique du Porc, Paris. 